\title{
Décadrages Décadrages
}

cinéma, à travers champs Cinéma, à travers champs

$43 \mid 2020$

Abdellatif Kechiche

\section{Une guerre sur les écrans? Le cinéma en Suisse et la Première guerre mondiale.}

Compte rendu du livre d'Adrian Gerber, Zwischen Propaganda und Unterhaltung. Das Kino in der Schweiz zur Zeit des Ersten Weltkriegs, Marburg, Schüren, 2017 (Zürcher Filmstudien 37)

Pierre-Emmanuel Jaques

\section{OpenEdition}

1 Journals

Édition électronique

URL : https://journals.openedition.org/decadrages/1608

DOI : 10.4000/decadrages. 1608

ISSN : 2297-5977

Éditeur

Association Décadrages

Édition imprimée

Date de publication : 1 juillet 2020

Pagination : 186-191

ISSN : 2235-7823

Référence électronique

Pierre-Emmanuel Jaques, « Une guerre sur les écrans ? Le cinéma en Suisse et la Première guerre

mondiale. », Décadrages [En ligne], 43 | 2020, mis en ligne le 15 octobre 2021, consulté le 04 avril 2022. URL : http://journals.openedition.org/decadrages/1608; DOI : https://doi.org/10.4000/decadrages. 1608 


\section{Zwischen} Propaganda und Unterhaltung

Das Kino in der Schweiz zur Zeit des Ersten Weltkriegs 
UNE GUERRE SUR LES ÉCRANS?

LE CINÉMA EN SUISSE ET

LA PREMIĖRE GUERRE MONDIALE

\author{
Compte rendu du livre d'Adrian Gerber, \\ Zwischen Propaganda und Unterhaltung. \\ Das Kino in der Schweiz zur Zeit des Ersten Weltkriegs, \\ Marburg, Schüren, 2017 (Zürcher Filmstudien 37)
}

Certaines publications bouleversent profondément la connaissance liée à tel ou tel aspect de I'histoire du cinéma: la thèse d'Adrian Gerber, parue sous la forme remaniée d'un livre de 620 pages, modifie fondamentalement notre connaissance de I'histoire du cinéma en Suisse jusqu'à la fin de la Première Guerre mondiale - et même audelà, dans la mesure où $A$. Gerber donne de précieuses indications sur les transformations affectant la branche cinématographique dans les années 1920. Jusque-là, les informations restaient incomplètes et étaient dispersées dans différentes publications plus ou moins approfondies, et pas toujours accessibles ${ }^{1}$. Notre savoir ne permettait pas de disposer d'un tableau satisfaisant de la période concernée, en particulier du moment de la Première Guerre. Si les études sur le cinéma des premiers temps ont connu un développement extraordinaire suite au colloque de Brighton de 1978 et grâce à l'implication de nombreux chercheurs, notamment au sein de l'association Domitor, elles restent néanmoins limitées du fait de la disparition de très nombreux documents. Et si plusieurs films considérés comme perdus ont pu être retrouvés, une très importante proportion des pellicules les plus anciennes ont définitivement disparu. II en va de même en Suisse, où la situation est renforcée par la taille et la durée plutôt limitées des premières entreprises de production. II faut attendre la création d'Eos-Film à Bâle en 1914 pour voir une société assurer une production régulière, notamment dans les domaines des actualités et des documentaires. Poursuivie jusque dans les années 1940 sous le nom d'Eoscop, cette activité se traduit sous la forme d'un laboratoire et d'un petit studio, repris par August Kern dans les années 1960, ce qui a permis à quelques titres produits par la firme bâloise d'arriver finalement à la Cinémathèque suisse. En ce qui concerne les documents non-film, le tableau ne diffère que peu: les entreprises sont restées de taille modeste et n'ont que rarement connu une continuité telle qu'elles aient conservé des documents remontant à la période des années 1910. Au plan administratif, I'absence de législation nationale a aussi contribué à limiter la constitution d'une documentation représentative du développement du cinéma dans tout le pays. C'est au
1 La somme d'Hervé Dumont, Histoire du cinéma suisse. Films de fiction 1896-1965 (Lausanne, Cinémathèque suisse, 1987) a permis l'établissement d'un premier canevas. Les travaux les plus riches sur cette période sont ceux de Roland Cosandey, notamment dans sa synthèse: "Le cinéma en Suisse: les vingt premières années (1895-1914)", dans Rémy Pithon (éd.), Cinéma suisse muet, lumières et ombres, Lausanne, Antipodes/ Cinémathèque suisse, 2002, pp.33-40. Nous avions proposé quelques pistes avec Gianni Haver, Le Spectacle cinématographique en Suisse 1895-1945, Lausanne, Antipodes, 2003. 
2 Konrad J. Kuhn, Béatrice Ziegler (éd.), Der vergessene Krieg. Spuren und Traditione zur Schweiz im Ersten Weltkrieg, Baden, Hier und jetzt, 2014.

3 On peut citer: Roman Rossfeld, Thomas Buomberger, Patrick Kury (éd.), 14/18. La Suisse et la Grande Guerre, Baden, Hier und jetzt, 2014; Christophe Vuilleumier (éd.), La Suisse et la guerre de 1914-1918: actes du colloque tenu du 10 au 12 septembre 2014 au Château de Penthes, Genève, Slatkine, 2015. Plusieurs autres études ont été menées, dont parmi les plus riches: Regula Schmid Keeling, Peter Niederhäuser, Erika Hebeisen (éd.), Kriegs- und Krisenzeit. Zürich während des Ersten Weltkriegs, Zurich, Chronos, 2014 - dans lequel A. Gerber a publié un article.

$4 \quad$ Alexandre Elsig, Les Shrapnels du mensonge, Lausanne, Antipodes, 2017. Idem, pp.370-383.

$6 \quad$ Une version noir-blanc a été mise en ligne à l'initiative de la Bibliothek am Guisanplatz: https://www.big.admin.ch Le film vient d'être restauré par la Cinémathèque suisse, qui s'est efforcée de dupliquer au mieux les teintages et virages présents à l'époque. plan cantonal et local que l'on trouve certains renseignements mais souvent limités à des demandes d'ouvertures de salles et lors de changements législatifs (interdiction des enfants dans la plupart des cantons vers 1915; instauration de censures cantonales; réglementation concernant l'ouverture de cinémas; etc.). La branche cinématographique tendra à s'organiser dès le début des années 1910, donnant lieu à l'édition en 1911 d'un hebdomadaire, Kinema, mais dont les premiers numéros conservés ne remontent qu'à 1913.

Par ailleurs, les publications liées aux salles (affiches, programmes et autres ephemera) n'ont quasi jamais été prises en compte par les institutions archivistiques et ont donc très largement disparu. Cette situation explique certainement le caractère lacunaire des études publiées jusqu'alors et dont la grande majorité consistait en des monographies locales, souvent réalisées sous la forme de mémoires universitaires consacrés à l'une ou l'autre région.

Moins directement touchée par le conflit armé que les autres pays européens, la Suisse entretient un rapport mémoriel (et émotionnel) plus distancé avec ce passé ${ }^{2}$. Néanmoins, le centenaire de la Première Guerre a suscité un renouvellement historiographique considérable. Faisant suite à une importante exposition organisée avec le Musée national suisse - ou plutôt devrait-on dire une série d'expositions -, différents colloques ont passé en revue les aspects militaires, économiques, sociaux, politiques et culturels de la Suisse pendant cette période difficile. Si le fossé entre la Suisse alémanique et romande peut paraître de moindre ampleur qu'envisagé auparavant, les chercheurs insistent à présent sur la fracture sociale qui déboucha sur la Grève générale de 1918, ainsi que sur certains changements de paradigme économique, la Suisse devenant une place financière importante durant cette période. Plusieurs volumes collectifs sont parus, attestant d'un profond renouvellement, tant en termes de questionnement, de méthode, que de connaissances. ${ }^{3}$ Parmi les études innovantes, la thèse d'Alexandre Elsig, Les Shrapnels du mensonge, s'est imposée comme une des contributions incontournables concernant le rôle de la propagande déployée en Suisse par les nations belligérantes ${ }^{4}$. Soucieuses de s'allier les populations des pays neutres, les nations en guerre cherchent à gagner leur faveur par une intense propagande, dans la presse, les spectacles et la culture en général. A. Elsig consacre un sous-chapitre à la question du cinéma, "La guerre dans les salles obscures ${ }^{5}$. À ses yeux, I'Allemagne et la France contrôleraient un nombre important de cinémas, mais l'effet de la propagande serait néanmoins limité, tout en attestant du fossé entre Alémaniques et Romands. La mise en place d'une production cinématographique locale, avec notamment les films Der Bergführer (Eduard Bienz, production Schweizer Express-Films, 1917) et L'Armée suisse (production Eos-Film, 1918) ${ }^{6}$ lui apparaissant comme une réaction à la présence de films étrangers marqués par un caractère propagandiste évident, avant tout allemands et français. Ses observations reposent sur les documents émis par le Comte Harry Graf Kessler, chargé de la propagande culturelle au sein de la Légation allemande 
et dont le journal a été édité7. II s'appuie aussi sur les nombreux rapports adressés à la Zentralstelle für Auslandsdienst, centrale de propagande à l'étranger, à Berlin, ainsi que de nombreux documents conservés aux Archives fédérales (à Berne) liés au Département politique (aujourd'hui Département des affaires étrangères). II mobilise enfin la presse, notamment le Journal de Genève, facilement accessible en ligne, et dont on sait l'écho important qu'il eut à l'étranger pendant le conflit.

Si Adrian Gerber partage certaines hypothèses avec Elsig, son travail s'en éloigne cependant considérablement et questionne certaines des données rassemblées par l'historien fribourgeois. II axe sa recherche sur une méthodologie historique qualifiée d'"historische Medienwahrnemung", c'est-à-dire qui cherche à situer la place du cinéma dans la société et à analyser la réception, en contexte, des films par des publics déterminés - tout en examinant leur réaction quand cela est possible. Forte d'une tradition philosophique qui se base sur le concept de sphère publique (Habermas), ce type de recherche sur la "Kinoöffentlichkeit" a suscité des travaux d'importance comme ceux de Miriam Hansen, aux États-Unis ${ }^{8}$, et de Corinna Müller, en Allemagne ${ }^{9}$, prolongés par différentes études notamment au sein du Seminar für Filmwissenschaft ${ }^{10}$, où Adrian Gerber a effectué cette thèse, dirigée par Margrit Tröhler et Jörg Schweinitz, et publiée dans la série des Zürcher Filmstudien. En liant des aspects matériels à une problématique plus théorique, cette démarche assure une profondeur aux analyses effectuées. Se fondant sur des éléments très concrets, tirés de sources dont la validité est sans relâche éprouvée, la présente recherche articule ainsi une interprétation de portée générale avec des points de détail qui sont situés dans un contexte social, politique, économique et culturel. Pour mener à bien sa recherche, Adrian Gerber s'est trouvé, comme la plupart des chercheurs et chercheuses intéressé·e·s à cette période, confronté à une carence de sources. II a donc procédé en analysant dans le détail un périodique comme Kinema ${ }^{11}$, tout en s'intéressant à de nombreuses sources souvent inédites (notamment des articles dans des publications régionales, des mémoires de licence, etc.) et en découvrant, aux Archives fédérales et dans des archives locales, une masse de documents jusqu'alors ignorés. A. Gerber a ainsi réussi à rassembler un ensemble remarquable de données, tout en sachant les pondérer en les confrontant les unes aux autres. L'historien a aussi découvert des fonds qui avaient échappé jusqu'alors aux chercheur·euse·s l'ayant précédé. Dans le bâtiment qui abrita l'une des plus anciennes salles de Zurich, le Radium, ont été découvertes une série d'affiches qui donnent une idée de la manière dont les films ont pu apparaître sur les nombreux lieux d'affichages annonçant ses riches programmes $^{12}$. Tout en disant se concentrer prioritairement sur Zurich, A. Gerber nous donne en fait le tableau du développement des cinémas en Suisse de 1906 - année d'ouverture de la première salle permanente du pays, à Genève - jusqu'à 1920, en listant précisément toutes les salles, leurs propriétaires, etc. II retrace ensuite la mise en
7 Günter Riederer (éd.), Harry Graf Kessler, Das Tagebuch 1880-937. Band 6: 19161918, Stuttgart, Klett-Cotta, 2006.

8 Miriam Hansen, Babel and Babylon. Spectatorship in American Silent Film, Cambridge, Harvard University Press, 1991.

9 Corinna Müller, Frühe deutsche Kinematographie: Formale, wirtschaftliche und kulturelle Entwicklungen 1907-1912, Stuttgart, Weimar, Metzler, 1994.

10 Voir notamment Irmbert Schenk, Margrit Tröhler, Yvonne Zimmermann (éd.), Film - Kino - Zuschauer:

Filmrezeption/Film - Cinema Spectator: Film Reception, Marburg, Schüren, 2010.

11 Il faut souligner d'ailleurs que c'est grâce à l'insistance d'Adrian Gerber et du Seminar für Filmwissenschaft que la collection conservée par la Bibliothèque nationale, à Berne, a été numérisée et mise en ligne, ainsi que d'autres publications spécialisées. Voir les sites: https://www.film.uzh.ch et https://www.e-periodica.ch 12 Voir https://www.cinematheque.ch 
place du système de location, dit de monopole, qui s'établit progressivement en Suisse. Pour ce faire, Gerber a trouvé un fonds remarquable, conservé à la Dokumentationsstelle (Cinémathèque suisse) à Zurich: les archives du distributeur Monopol-Films L. Burstein qui donnent une image très concrète de la diffusion des films acquis auprès de sociétés étrangères (nombre de copies, durée d'exploitation, sous-location, etc.). Dans le contexte d'une évaluation de la place du cinéma dans la propagande déployée en Suisse par les belligérants, la précision des données ainsi rassemblées permet de corriger certaines inexactitudes: si certaines sociétés relaient en effet des sociétés allemandes, comme Nordisk, qui dépend de la filiale berlinoise d'une société à l'origine danoise, elles sont restées pour la plupart indépendantes et se sont d'abord pliées au jeu commercial de proposer des films à des salles elles aussi concernées en premier lieu par la rentabilité. Et si l'on voit que certaines légations ont bien cherché à influencer le jeu normal des échanges, A. Gerber démontre aussi que leur effet est finalement resté limité. On peut citer, à titre d'exemple, l'anecdote suivante: pour éviter la trop grande diffusion du film Mères françaises, un "drame patriotique" avec Sarah Bernhardt insistant sur la souffrance des Françaises et montrant les dégâts affectant la Cathédrale de Reims, le Comte Harry Kessler a été chargé d'acheter la copie du film circulant dans le pays - ce qu'il arrive à faire sans grande difficulté -, mais que cela n'empêcha pas le film de Mercanton et Richepin de circuler très rapidement à nouveau dans les salles suisses...

Mais, et cela méritait d'être clairement posé, la propagande nationaliste, qu'elle provienne des pays de l'Entente ou des Empires centraux, s'est concentrée dans les actualités et les documentaires. Cette part est d'ailleurs particulièrement difficile à approcher car les bandes diffusées en Suisse ont pour l'essentiel purement et simplement disparu. Quelques exemples conservés en France, en Allemagne ou en Italie permettent de se faire une idée du type d'images en présence mais sans que nous puissions être assurés qu'elles correspondent à ce qui a été effectivement projeté sur les écrans suisses. On peut donc se demander si, parfois, certains sujets ont été édités spécialement pour la Suisse ou non.

Parmi les analyses particulièrement probantes, je retiendrai celle du film Graf Dohna und seine Möwe (prod. BUFA, 1917), un film consacré à la guerre sur les mers qui voit des navires alliés coulés par un navire allemand. Si le film a rencontré un succès certain en Suisse, le consulat zurichois s'inquiète de la réaction du public qui ne semblait que peu apprécier le coulage des navires, surtout quand il s'agissait de bâtiments civils...

Parfois, à l'occasion de certaines batailles, les deux blocs en présence ont poursuivi la guerre sur les écrans suisses. Les films pris à I'occasion de la dixième bataille de I'Isonzo (1918) diffèrent largement lorsqu'ils proviennent soit de la société viennoise Sascha-Film, soit d'Italie (une production Comerio - Sezione Cinematografica del Regio Esercito). Attestant aussi des divisions en Suisse, le premier 
est montré en Suisse alémanique alors qu'il n'est pas projeté en Romandie. Le film italien passe lui dans tout le pays. Leur accueil dans la presse montre aussi à quel point les critiques s'efforcent pour la plupart d'éviter de prendre parti, soulignant plutôt la violence des combats et l'authenticité des images. Pour effectuer de telles analyses, A. Gerber a su identifier des publications jusqu'alors ignorées par la recherche.

Analysant quelques films présentant les internés en Suisse, A. Gerber a découvert l'existence, outre des sujets français ou allemands, d'un film suisse destiné à affirmer la neutralité du pays et à souligner son engagement humanitaire: Die Durchreise des französischen Evakuierten durch die Schweiz (1918). S'il reste à mes yeux des questions sur l'identification des différents films conservés, notamment celui à la Cinémathèque suisse, ceux conservés à I'Imperial War Museum à Londres et à l'Établissement de communication et de production audiovisuelle de la Défense à Ivry laissent clairement apparaître une volonté de présenter la Suisse comme un pays de tradition humanitaire désireux d'apporter une forme de réconfort aux victimes de la guerre.

Aussi, grâce à une analyse fine des documents et à leur contextualisation, Adrian Gerber livre ici une étude de premier plan, capable de saisir la réception des films en Suisse dans un contexte transnational qui montre avec précision l'insertion de la Suisse dans un vaste marché international et au carrefour des luttes qui affectent le territoire européen. Soulignons enfin que les illustrations sont fort bien reproduites et qu'elles rendent compte avec précision de la qualité visuelle des copies teintées de l'époque. Regrettons toutefois que l'éditeur n'ait pas jugé utile d'établir un index, tout en nous rappelant que l'ouvrage, également disponible en open access, permet de rechercher sous cette forme les noms et autres titres dans les fichiers ainsi mis à disposition des intéressé·e·s... 